07

\title{
Экспериментальное исследование тонких пленок твердого электролита фосфор-оксинитрида лития
}

(C) A.C. Рудый ${ }^{1,2}$, С.В. Васильев ${ }^{1,2}$, М.Е. Лебедев ${ }^{2}$, А.В. Метлицкая ${ }^{1,2}$, А.А. Мироненко ${ }^{2}$, В.В. Наумов ${ }^{2}$, А.В. Новожилова ${ }^{1,2}$, И.С. Федоров ${ }^{2}$, А.Б. Чурилов ${ }^{1,2}$

${ }^{1}$ Ярославский государственный университет им. П.Г. Демидова, Ярославль

${ }^{2}$ Ярославский филиал Физико-технологического института РАН,

Ярославль

E-mail: rudy@uniyar.ac.ru

Поступило в Редакцию 27 декабря 2016 г.

Представлены результаты исследования тонкопленочных образцов твердого электролита LiPON, полученных методом магнетронного нанесения. Приведены данные о морфологии, структуре, элементном и фазовом составе пленок LiPON и результаты исследования их электрофизических параметров.

DOI: 10.21883/PJTF.2017.11.44690.16637

Одним из направлений разработки литий-ионных аккумуляторов (ЛИА) является их миниатюризация и переход на твердотельные ЛИА (в англоязычной литературе all solid-state lithium-ion battery). На этом пути возникает ряд новых технических задач, одна из которых состоит в нанесении твердого электролита. Очевидно, что технология нанесения электролита должна содержать как можно меньше операций, а для определенного класса твердотельных ЛИА она должна быть и СБИС-совместимой. Из твердых электролитов этим требованиям наилучшим образом отвечает фосфор-оксинитрид лития (LiPON), полученный более 20 лет назад [1-4] в Oak Ridge National Laboratory. 
Последний обычно наносится в виде тонкой пленки толщиной $\sim 1 \mu \mathrm{m}$ магнетронным распылением ортофосфата лития в разреженной атмосфере азота.

Хотя в зарубежной научной периодике, например [3-9], приводятся параметры процесса нанесения LiPON, эти сведения далеко не полны. Кроме того, они индивидуальны для каждой установки и технологического маршрута. Поэтому при разработке технологии изготовления интегрального ЛИА задачи нанесения LiPON, изготовления тестовых структур и разработки методики их исследования приходится решать заново. Ниже приведены результаты исследования пленок LiPON, полученных методом магнетронного нанесения на установке SCR-651 „Tetra“.

Пленки LiPON наносились распылением мишени $\mathrm{Li}_{3} \mathrm{PO}_{4}$ диаметром $92.6 \mathrm{~mm}$ и толщиной $4.7 \mathrm{~mm}$. Мишень была изготовлена из порошка $\mathrm{Li}_{3} \mathrm{PO}_{4}$ методом градиентного прессования с последующим отжигом. Для предотвращения растрескивания мишень закреплялась в медный держатель, обеспечивающий равномерное распределение температуры и эффективный теплоотвод при распылении.

Основная задача на этапе отработки режимов нанесения LiPON методом магнетронного распыления состояла в получении аморфной структуры, поскольку кристаллический LiPON имеет низкую ионную проводимость. Для экспериментальных исследований пленок LiPON были изготовлены несколько партий структур двух видов:

- LiPON $600 \mathrm{~nm} / \mathrm{Pt} 100 \mathrm{~nm} / \mathrm{Ti} 10 \mathrm{~nm} /\left(\mathrm{SiO}_{2} / \mathrm{Si}\right)$;

- Pt $100 \mathrm{~nm} / \mathrm{LiPON} 600 \mathrm{~nm} / \mathrm{Pt} 100 \mathrm{~nm} / \mathrm{Ti} 10 \mathrm{~nm} /\left(\mathrm{SiO}_{2} / \mathrm{Si}\right)$.

Здесь в скобках указана подложка, а последовательность слоев в строке соответствует направлению „сверху вниз“. Площадь поверхности платиновых контактов составляла $2 \times 2 \mathrm{~cm}^{2}$.

Пленки LiPON исследовались методами рентгеновской дифрактометрии, электронной сканирующей микроскопии, энергодисперсионного анализа и потенциометрии. Дифрактограммы пленок LiPON, полученные на рентгеновском дифрактометре ARL X'tra $(30 \mathrm{kV}, 30 \mathrm{~mA}$, $\left.\mathrm{Cu} K_{\alpha}\right)$, свидетельствуют об аморфной структуре пленок. На них отсутствуют какие-либо пики (в том числе ортофосфата лития), за исключением рефлексов от кремниевой подложки.

Морфология пленок LiPON исследовалась на сканирующем электронном микроскопе Supra-40. Исследования морфологии показали, что пленки LiPON получаются глянцевыми, похожими на пленки $\mathrm{SiO}_{2}$.

Письма в ЖТФ, 2017, том 43, вып. 11 
Результаты энергодисперсионного анализа пленки LiPON без учета Li

\begin{tabular}{c|c|c|c|c}
\hline Элемент & Линия & $\begin{array}{c}\text { Массовая } \\
\text { доля } \\
\text { элемента, \% }\end{array}$ & $\begin{array}{c}\text { Погрешность } \\
\text { массовой } \\
\text { доли } \sigma, \%\end{array}$ & $\begin{array}{c}\text { Атомная доля } \\
\text { элемента, \% }\end{array}$ \\
\hline $\mathrm{N}$ & $K_{\alpha 1 \_2}$ & 4.41 & 0.23 & 6.24 \\
$\mathrm{O}$ & $K_{\alpha 1-2}$ & 54.37 & 0.18 & 67.38 \\
$\mathrm{P}$ & $K_{\alpha 1}$ & 41.22 & 0.16 & 26.38
\end{tabular}

По данным электронной микроскопии латеральные размеры неоднородностей пленок не превышают $200 \mathrm{~nm}$, а шероховатость поверхности $-50 \mathrm{~nm}$.

Элементный состав наиболее интересных участков пленок LiPON изучался на энергодисперсионной приставке INCAx-act к микроскопу Supra-40. В таблице приведен автоматический расчет химического состава пленок LiPON без учета лития, поскольку он недоступен для наблюдения данным методом. Результаты измерений нормированы следующим образом: сумма всех элементов, кроме лития, равна $100 \%$.

Эквивалентная схема исследуемого образца (рис. 1) представляет собой два индукционно-связанных электрических слоя, соединенных сопротивлением $R_{I n t}$. Структура слоя потенциалопределяющих ионов (см. вставку на рис. 1) во многом схожа со строением двойного электрического слоя, поэтому далее для обозначения емкости в эквивалентной схеме используется термин „двойной электрический слой“ ${ }^{\circ}(\mathrm{EDL})$.

Для измерения электрофизических и емкостных характеристик LiPON, таких как емкость двойного электрического слоя $C_{\text {EDL }}$, внутреннее сопротивление $R_{\text {Int }}$ и время релаксации поляризации $\tau_{R}$, использовалось устройство, принципиальная схема которого показана на рис. 1. В процессе измерения регистрировались зарядные $I(t)$ и разрядные $U(t)$ кривые. Электрофизические характеристики структуры Pt $100 \mathrm{~nm} / \mathrm{LiPON} 600 \mathrm{~nm} / \mathrm{Pt} 100 \mathrm{~nm} / \mathrm{Ti}$ $10 \mathrm{~nm} /\left(\mathrm{SiO}_{2} / \mathrm{Si}\right)$ (далее просто $\mathrm{Pt} / \mathrm{LiPON} / \mathrm{Pt}$ ) определялись путем подгонки под экспериментальные данные параметров аппроксимирующей

Письма в ЖТФ, 2017, том 43, вып. 11 


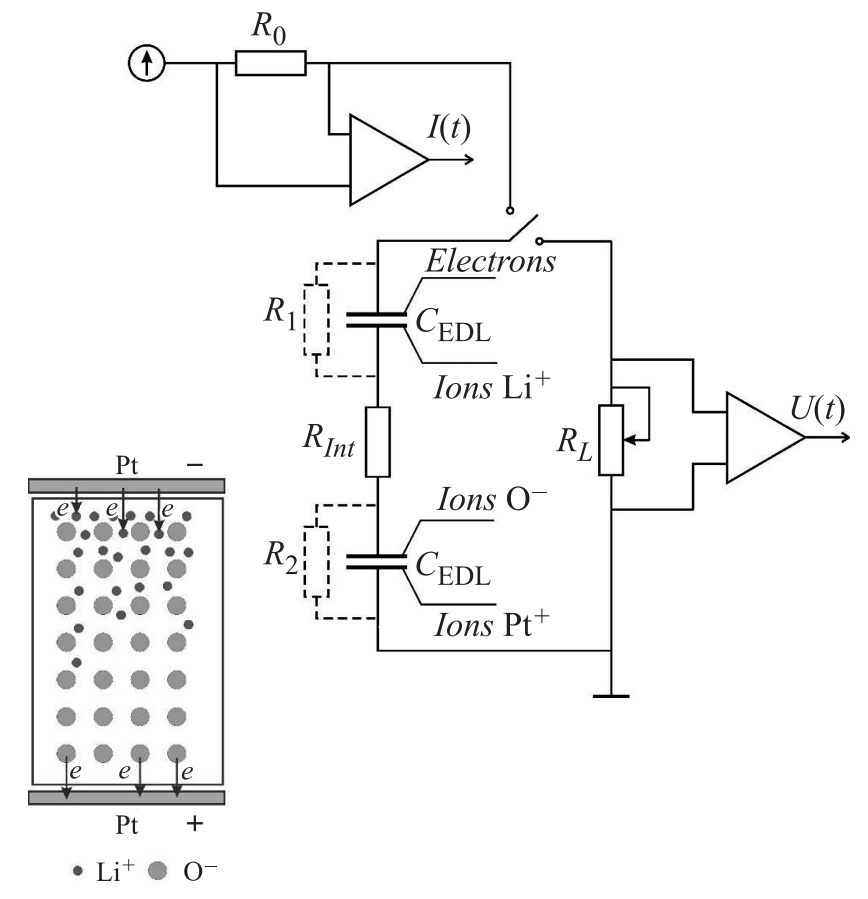

Рис. 1. Эквивалентная схема измерения разряда структуры Pt $100 \mathrm{~nm} / \mathrm{LiPON}$ $600 \mathrm{~nm} / \mathrm{Pt} 100 \mathrm{~nm} / \mathrm{Ti} 10 \mathrm{~nm}$ на $\mathrm{SiO}_{2} / \mathrm{Si} . R_{0}=100 \mathrm{k} \Omega, R_{\text {Int }}$ - сопротивление слоя LiPON, $R_{L}=0.1 \mathrm{M} \Omega-$ сопротивление нагрузки, $C_{\mathrm{EDL}}-$ емкость двойного электрического слоя. На вставке слева показан предполагаемый механизм релаксации поляризации LiPON: маленькие кружки - ионы лития, большие ионы кислорода.

зависимости

$$
U=U_{0}\left(1+\alpha t / \tau_{0}\right)^{-1 / \alpha} e^{-1 / \tau_{D}}
$$

где

$$
\tau_{D}=\left(R_{\text {Int }}+R_{L}\right) C_{\mathrm{DEL}} / 2
$$

- время релаксации поляризации за счет процессов переноса (дрейфовый и диффузионный токи).

Письма в ЖТФ, 2017, том 43, вып. 11 
Зависимость (1) является решением начальной задачи, моделирующей процесс разряда структуры $\mathrm{Pt} / \mathrm{LiPON} / \mathrm{Pt}$ через нагрузочное сопротивление $R_{L}$. При построении начальной задачи скорость рекомбинации носителей заряда, образующих двойной электрический слой, полагалась зависящей от времени и описывалась в приближении времени релаксации. Предполагалось, что время релаксации $\tau_{R}$ растет вследствие снижения концентрации потенциалопределяющих ионов в слое Гельмгольца в результате их рекомбинации. Рост времени релаксации аппроксимировался линейной зависимостью

$$
\tau_{R}(t) \approx \tau_{0}+\alpha t
$$

Поскольку процесс рекомбинации связан с переносом заряда и диссипацией энергии и импульса электронов на интерфейсах Pt/LiPON, на эквивалентной схеме ему соответствует ток утечки через резисторы $R_{1}$ и $R_{2}$.

Для определения параметров решения (1) регистрировались зарядные и разрядные кривые структуры Pt/LiPON/Pt. Ha первом этапе исследуемая структура заряжалась до потенциала $1 \mathrm{~V}$ током $I \leqslant 10 \mu \mathrm{A}$ и регистрировалась зависимость $I(t)$, по которой вычислялись заряд $Q(t)$ (рис. 2) и удельная зарядная емкость структуpы Pt/LiPON/Pt. При этом было получено значение полного заряда $Q=1.91 \cdot 10^{-5} \mathrm{C}$, которое хорошо согласуется со значением $Q=4 \cdot 10^{-9} \mathrm{~A} \cdot \mathrm{h} / \mathrm{cm}^{2}$, полученным для того же образца на многоканальном потенциостат-гальваностате Elins P-20X8 в потенциальном окне $0-4 \mathrm{~V}$. Отметим, что зарядная емкость $Q=1.91 \cdot 10^{-5} \mathrm{C}$ при потенциале $U=1 \mathrm{~V}$ численно равна емкости двойного электрического слоя $C_{\mathrm{EDL}}$. В простейшей модели Гельмгольца последняя рассчитывается как емкость плоского конденсатора $C_{\mathrm{EDL}}=\varepsilon_{0} S / d$, где $d$ - величина порядка диаметра потенциалопределяющего иона. При площади обкладок конденсатора $S=4 \cdot 10^{-4} \mathrm{~m}^{2}$ экспериментальному значению электрической емкости $C_{\mathrm{EDL}}=1.91 \cdot 10^{-5} \mathrm{~F}$ соответствует $d=1.85 \cdot 10^{-10} \mathrm{~m}$. Для сравнения: диаметр иона лития составляет $1.56 \cdot 10^{-10} \mathrm{~m}$.

На втором этапе измерений регистрировался разряд через прецизионное переменное сопротивление $R_{L}$. Параметры выражения (1) определялись путем его подгонки к экспериментальным кривым методом наименьших квадратов по алгоритму Левенберга-Марквардта.

Письма в ЖТФ, 2017, том 43, вып. 11 


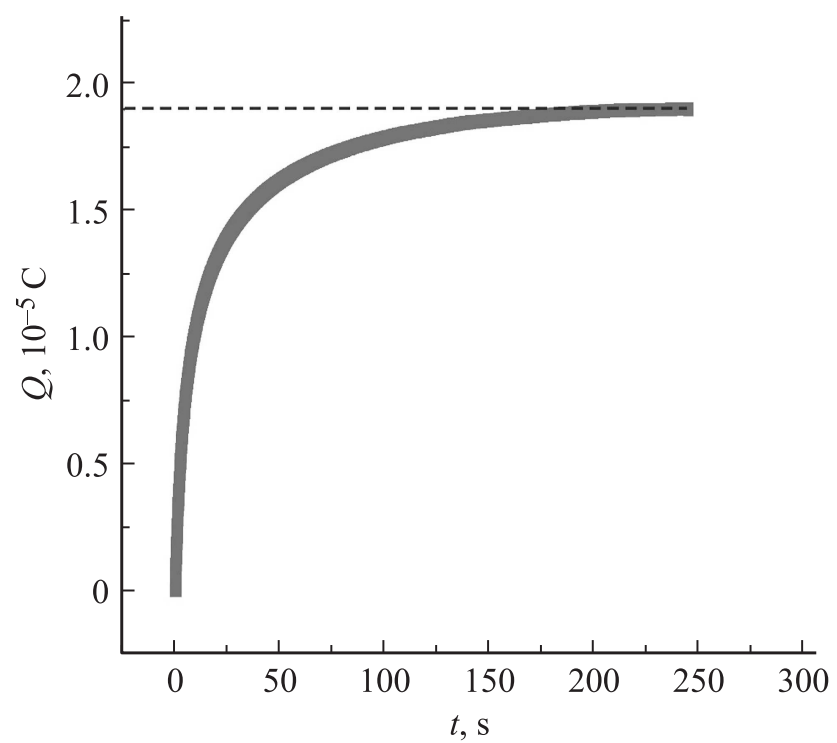

Pис. 2. График зависимости заряда структуры Pt/LiPON/Pt от времени при зарядке до потенциала $1 \mathrm{~V}$.

Измерения показали, что на графиках зависимости $\ln U(t)$ при больших сопротивлениях нагрузки четко видны участки гиперболической (в области малых $t$ ) и линейной (в области больших $t$ ) зависимостей от времени. Для малых сопротивлений нагрузки, как и ожидалось, наблюдается только гиперболическая зависимость от времени. Совпадение экспериментальных и подгоночных разрядных кривых иллюстрирует рис. 3, на котором показана зависимость напряжения от времени при разряде через сопротивление $1 \mathrm{k} \Omega$.

Задача определения параметров разряда состояла в обнаружении порогового значения $R_{L}$, после которого скорость разряда будет ограничиваться сопротивлением LiPON, т. е. $R_{\text {Int }}$. Основным результатом исследования разрядных кривых является отсутствие влияния $R_{\text {Int }}$ вплоть до значения $R_{L}=10 \Omega$. Так как при малых значениях сопротивления нагрузки резко ухудшается соотношение сигнал/шум, более точное определение порогового значения $R_{L}$ не 


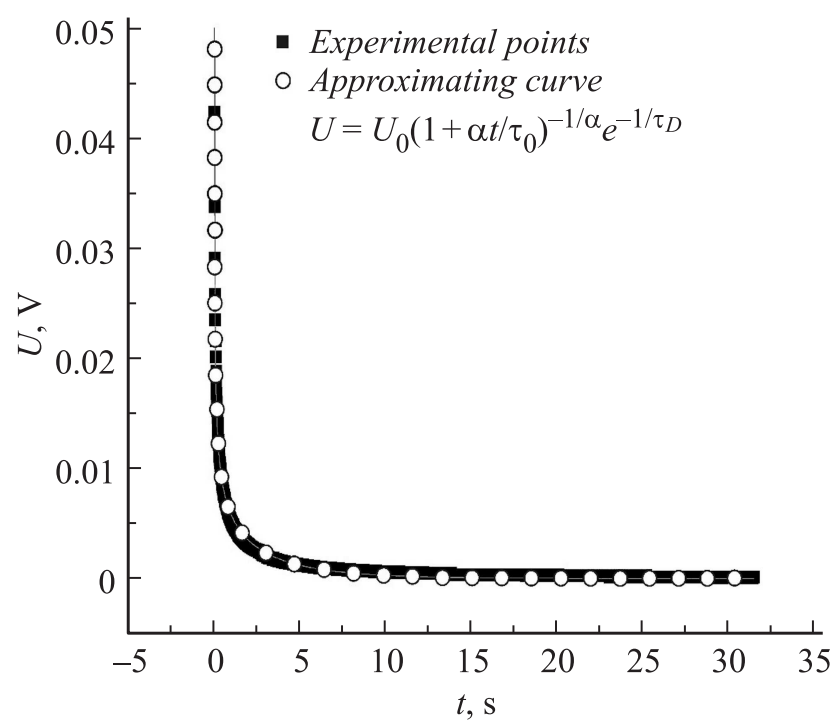

Рис. 3. Экспериментальная и теоретическая зависимости напряжения на электродах структуры Pt/LiPON/Pt от времени при разряде через сопротивление $1 \mathrm{~kW}$.

представляется возможным. Здесь необходимо пояснить, что при испытании аккумуляторных структур режим разряда обычно лежит в интервале $1-10 \mathrm{C}$, при этом режим $10 \mathrm{C}$ считается „жестким“ для ЛИА с жидким электролитом. При удельной емкости структуры $Q=4 \cdot 10^{-9} \mathrm{~A} \cdot \mathrm{h} / \mathrm{cm}^{2}$ и площади $4 \mathrm{~cm}^{2}$ режиму разряда $1 \mathrm{C}$ соответствует ток разряда $I=16 \mathrm{nA}$. Тогда средний ток разряда через сопротивление $100 \Omega$, определяемый как $I=Q / 3 \tau_{D}$, с учетом соотношения $\tau_{D}=R_{L} Q / 2$ составит $6.67 \mathrm{~mA}$, что соответствует режиму $4 \cdot 10^{5} \mathrm{C}$.

Исходя из соотношения $R_{I n t} \sim 10 \Omega$, удельное сопротивление LiPON можно оценить как $\rho \sim 6.6 \cdot 10^{5} \Omega \cdot \mathrm{cm}$, а проводимость как $\sigma \sim 1.5 \cdot 10^{-6} \mathrm{~S} \cdot \mathrm{cm}^{-1}$, что совпадает с данными работы [4]. Для получения более точных значений проводимости необходимо изменить топологию образца, однако это неизбежно приведет к снижению точности измерения его емкостных характеристик.

Письма в ЖТФ, 2017, том 43, вып. 11 
Концентрация подвижных ионов лития для полученных значений зарядной емкости при толщине пленки $600 \mathrm{~nm}$ составляет $n_{\mathrm{Li}}=1.50 \cdot 10^{18} \mathrm{~cm}^{-3}$. При этом подвижность ионов лития на основании приведенной выше оценки проводимости может быть оценена как $\mu>6.5 \cdot 10^{-6} \mathrm{~cm}^{2} / \mathrm{V} \cdot \mathrm{s}$, а соответствующее значение коэффициента диффузии - как $D>1.6 \cdot 10^{-7} \mathrm{~cm}^{2} / \mathrm{s}$.

Резюмируя сказанное, можно утверждать, что технология нанесения пленок LiPON методом высокочастотного магнетронного распыления, отработанная для установки SCR-651 Tetra, позволяет воспроизводимо получать пленки LiPON и изготавливать на их основе структуры с требуемыми характеристиками. Полученные экспериментальные образы LiPON по своему составу, морфологии и электрофизическим параметрам отвечают требованиям, предъявляемым к твердому электролиту для интегральных ЛИА. Совпадение экспериментальных кривых и аппроксимирующих зависимостей свидетельствует об адекватности предложенной модели разряда LiPON. Для повышения точности измерения сопротивления пленок LiPON и/или контроля качества твердого электролита необходимо дальнейшее усовершенствование методики измерений. Например, одновременно со структурой $\mathrm{Pt} / \mathrm{LiPON} / \mathrm{Pt}$ можно наносить тестовую структуру в виде полоски с контактными площадками и измерять ее сопротивление в продольном направлении.

Работа выполнена при финансовой поддержке Министерства образования и науки РФ, соглашение № 14.574.21.0099 от 26 августа 2014 г., уникальный идентификатор прикладных научных исследований (проекта) RFMEFI57414X0099.

\section{Список литературы}

[1] Bates J.B., Dudney N.J., Gruzalski G.R. et al. // Solid State Ion. 1992. V. 53-56. P. 647.

[2] Bates J.B., Dudney N.J., Gruzalski G.R. et al. // Power Sources. 1993. V. 43-44. P. 103.

[3] Xiaohua Yu., Bates J.B., Jellison G.E. // Proceedings of the Symposium on Thin Film Solid Ionic Devices and Materials. 1995. V. 95-22. P. 23.

[4] Xiaohua Yu., Bates J.B., Jellison G.E., Hart F.X. // Electrochem. Soc. 1997. V. 144. N 2. P. 524. 
[5] Ho Young Park, Sang Cheol Nam, Young Chang Lim et al. // Electroceram. 2006. V. 17. P. 1023.

[6] Zongqian Hu, Dezhan Li, Kai Xie // Bull. Mater. Sci. 2008. V. 31. N 4. P. 681.

[7] Nam-Seok Roh, Sang-Dong Lee, Hyuk-Sang // Scripta Mater. 2000. V. 42 . P. 43.

[8] Yoon Gu Kima, Wadley H.N.G. // J. Vac. Sci. Technol. 2008. V. 26. N 1. P. 174.

[9] Belous A.G., V'yunova O.I., Kovalenko L.L. et al. // Russ. J. Electrochem. 2014. V. 50. N 6. P. 523.

Письма в ЖТФ, 2017, том 43, вып. 11 